\title{
Splitting on Demand in SAT Modulo Theories
}

\author{
Clark Barrett $^{\star}$, Robert Nieuwenhuis ${ }^{\star \star}$, Albert Oliveras ${ }^{\star \star}$, and Cesare Tinelli ${ }^{\star \star \star}$
}

\begin{abstract}
Lazy algorithms for Satisfiability Modulo Theories (SMT) combine a generic DPLL-based SAT engine with a theory solver for the given theory $T$ that can decide the $T$-consistency of conjunctions of ground literals. For many theories of interest, theory solvers need to reason by performing internal case splits. Here we argue that it is more convenient to delegate these case splits to the DPLL engine instead. The delegation can be done on demand for solvers that can encode their internal case splits into one or more clauses, possibly including new constants and literals. This results in drastically simpler theory solvers. We present this idea in an improved version of $\operatorname{DPLL}(T)$, a general SMT architecture for the lazy approach, and formalize and prove it correct in an extension of Abstract DPLL Modulo Theories, a framework for modeling and reasoning about lazy algorithms for SMT. A remarkable additional feature of the architecture, also discussed in the paper, is that it naturally includes an efficient Nelson-Oppen-like combination of multiple theories and their solvers.
\end{abstract}

\section{Introduction}

The performance of propositional SAT solvers based on the Davis-PutnamLogemann-Loveland (DPLL) procedure $[9,8]$ has importantly improved during the last years, and DPLL-based solvers are becoming the tool of choice for attacking more and more practical problems. The DPLL procedure has also been adapted for handling problems in more expressive logics, and, in particular, for the Satisfiability Modulo Theories (SMT) problem: deciding the satisfiability of ground first-order formulas with respect to background theories such as the integer or real numbers, or arrays. SMT problems frequently arise in formal hardware and software verification applications, where typical formulas consist of very large sets of clauses like:

$$
p \quad \vee \quad \neg q \quad \vee \quad a=f(b-c) \quad \vee \quad \operatorname{read}(s, f(b-c))=d \quad \vee \quad a-g(c) \leq 7
$$

with propositional atoms as well as atoms over (combined) theories like the integers, arrays, or Equality with Uninterpreted Functions (EUF). SMT has become a very active area of research, and efficient SMT solvers exist that can handle (combinations of) many such theories $T$. Currently most SMT solvers follow the so-called lazy approach to SMT, combining (i) theory solvers that can

^ New York University, www.cs.nyu.edu/ barrett.

** Technical Univ. of Catalonia, Barcelona, www.lsi.upc.edu/ robertol oliveras. Partially supported by Spanish Ministry of Educ. and Science through the LogicTools project (TIN2004-03382, both authors), and FPU grant AP2002-3533 (Oliveras).

$\star \star \star$ Univ. of Iowa, www.cs.uiowa.edu/ ${ }^{\sim}$ inelli. Partially supp. by NSF grant 0237422 . 
handle conjunctions of literals over the given theory $T$, with (ii) DPLL engines for dealing with the Boolean structure of the formulas.

$\operatorname{DPLL}(T)$ is a general SMT architecture for the lazy approach [10]. It consists of a $\operatorname{DPLL}(X)$ engine, whose parameter $X$ can be instantiated with a $T$-solver Solver $_{T}$, thus producing a $\operatorname{DPLL}(T)$ system. The $\operatorname{DPLL}(X)$ engine always considers the problem as a purely propositional one. For example, if the theory $T$ is EUF, at some point $\operatorname{DPLL}(X)$ might consider a partial assignment containing, among many others, the four literals $a=b, f(a)=c, f(b)=d$, and $c \neq d$ without noticing its $T$-inconsistency, because it just considers such literals as propositional (syntactic) objects. But Solver $_{T}$ continuously analyzes the partial model that $\operatorname{DPLL}(X)$ is building (a conjunction of literals). It can warn $\operatorname{DPLL}(X)$ about this $T$-inconsistency, and generate a clause, called a theory lemma, like $a \neq b \vee f(a) \neq c \vee f(b) \neq d \vee c=d$, which can be used by $\operatorname{DPLL}(X)$ for backjumping. Solver ${ }_{T}$ sometimes also does theory propagation: as soon as, e.g., $\quad a=b, \quad f(a)=c$, and $f(b)=d$ become true, it can notify $\operatorname{DPLL}(X)$ about $T$-consequences like $c=d$ that occur in the input formula. The modular $\operatorname{DPLL}(T)$ architecture is flexible, and can be implemented efficiently: the BarcelogicTools implementation of $\operatorname{DPLL}(T)$ won all the four divisions it entered at the 2005 SMT Competition [1].

Here we propose an improved version of the DPLL $(T)$ architecture, to rationalize and simplify the construction of lazy SMT systems where Solver $_{T}$ does reasoning by cases. We present it formally by means of a corresponding extension of Abstract DPLL Modulo Theories, a uniform, declarative framework introduced in [12] for modeling and reasoning about lazy SMT procedures.

Example 1. In the array theory, the equation $\operatorname{read}(\operatorname{write}(A, i, v), j)=\operatorname{read}(A, j)$ holds in two situations: when the indices $i$ and $j$ are distinct, or when they are equal but the write $(A, i, v)$ changes nothing, i.e., the value of array $A$ at position $i$ is already $v$. Deciding the $T$-consistency of a large conjunction of equations and disequations over arrays essentially requires Solver $_{T}$ to do an analysis of many Boolean combinations of such cases. In the extension of DPLL $(T)$ we propose here, Solver $_{T}$ can delegate all such case splittings to the DPLL $(X)$ engine, e.g., it can demand $\operatorname{DPLL}(X)$ to split on atoms like $i=j$, by sending it a theory lemma (i.e., a ground clause valid in the theory) that encodes the split - for instance, a clause like $\operatorname{read}(\operatorname{write}(A, i, v), j) \neq \operatorname{read}(A, j) \vee i \neq j \vee \operatorname{read}(A, i)=v$.

The main novelty, and complication, versus the previous version of DPLL(T) is that the lemma may contain atoms that do not occur in the input formula. Sometimes even new constant symbols may be introduced. For example, in (fragments of) set theory [7], a set disequality $s \neq s^{\prime}$ may be handled by the theory solver by reducing it to the disjunction $\left(a \in s \wedge a \notin s^{\prime}\right) \vee\left(a \notin s \wedge a \in s^{\prime}\right)$, where $a$ is a fresh Skolem constant.

Centralizing all case splitting in the engine allows one to avoid the duplication of search functionality in the theory solver and drastically simplify its implementation, since a case splitting infrastructure is no longer necessary. Roughly, the solver's only requirement reduces to being able to detect $T$-inconsistencies once all case splits it has requested have been done. 
The main contribution of this paper is a general and formal specification of this sort of architecture, together with a rigorous proof of its correctness. The relevance of this architecture is that it unquestionably leads to simpler solvers for theories that require case splits - in practice, all theories $T$ where checking the $T$-inconsistency of ground literals is NP-hard.

In many SMT applications the background theory $T$ is defined as a combination of several component theories $T_{1}, \ldots, T_{n}$, each with its own local solver. An important aspect of our approach is that it can be naturally refined to accommodate such combined theories, giving rise to a $\operatorname{DPLL}\left(T_{1}, \ldots, T_{n}\right)$ architecture.

Example 2. Let $T$ be the union of two disjoint theories $T_{1}$ and $T_{2}$ where $T_{1}$ is EUF and $T_{2}$ is (some fragment of) arithmetic, two of the most common theories in SMT. Let $F$ be the conjunction $a=b \wedge f(a)-c \leq 3 \wedge f(b)-c \geq 4$ over the combined signature of $T_{1}$ and $T_{2}$. Introducing new constants $c_{1}$ and $c_{2}$, $F$ can be purified, into an equisatisfiable conjunction of the $T_{1}$-pure formula $F_{1}$ and the $T_{2}$-pure formula $F_{2}$ below:

$$
a=b \wedge c_{1}=f(a) \wedge c_{2}=f(b) \quad c_{1}-c \leq 3 \wedge c_{2}-c \geq 4 .
$$

In general, an arrangement $A$ for such pure conjunctions $F_{1} \ldots F_{n}$ is a conjunction saying, for every two constants shared between at least two different $F_{i}$ 's, whether the constants are equal or distinct. A general combination result underpinning the Nelson-Oppen method [11] states that for stably infinite and signature disjoint $T_{i}$ 's, $F$ is $T$-consistent if, and only if, for some arrangement $A$ each $F_{i} \wedge A$ is $T_{i}$-consistent (see, e.g., [14] for precise definitions and details). This can be decided by the respective $T_{i}$-solvers. In this example, $F$ is $T$-inconsistent since $F_{1} \wedge c_{1} \neq c_{2}$ is $T_{1}$-inconsistent and $F_{2} \wedge c_{1}=c_{2}$ is $T_{2}$-inconsistent.

In practice, it is useful if each $T_{i}$-solver is able to generate all clauses $c_{1}=$ $c_{1}^{\prime} \vee \ldots \vee c_{k}=c_{k}^{\prime}$ over the shared constants that are $T_{i}$-entailed by the conjunction $F_{i}$. For convex $T_{i}$, these entailed clauses are in fact always unit. It is not difficult to see that, if these two properties hold for all $T_{i}$, we only have to consider one arrangement: the one where every two constants not equated in a propagated equality are distinct. But usually the situation is less ideal. If some $T_{i}$ is nonconvex, it is necessary to do case splitting over the $T_{i}$-entailed non-unit clauses, and if some $T_{i}$-solver has limited or too expensive generation capabilities, the possible arrangements need to be (partially) guessed and tried.

By centralizing case splitting into the $\operatorname{DPLL}(X)$ engine and extending it to equalities over shared constants we can use the engine, in effect, to efficiently enumerate the arrangements on demand, that is, as requested by the individual theory solvers. Note that in the resulting $\operatorname{DPLL}\left(T_{1}, \ldots, T_{n}\right)$ architecture, the engine will again handle literals possibly not in the input clauses, namely, (dis)equalities between shared variables.

Section 2 of this paper introduces and discusses the correctness of an Extended Abstract DPLL Modulo Theories framework that formalizes our approach. ${ }^{1}$ Section 3 illustrates how to use the framework to avoid internal case

\footnotetext{
${ }^{1}$ Because of space constraints we cannot provide the correctness proof here. The complete proof can be found in [2].
} 
splits in a very general class of theory solvers. Section 4 discusses the application of the framework to $\operatorname{DPLL}\left(T_{1}, \ldots, T_{n}\right)$. Finally, Section 5 concludes.

Related work. Some of the ideas formalized in this paper on centralizing case splits in the Boolean engine are implemented in the system CVC [5] and CVCLite [4]. But apart from a brief note in Clark Barrett's PhD thesis (in Section 3.5.1 of [3]), we are not aware of any other description of them in the literature.

Bozzano et al. propose in [6] to use the Boolean engine in multi-theory SMT systems to do case splitting over the space of all possible arrangements. In contrast to this work, there the centralization of case-splitting concerns only equalities between shared constants, as needed by the Nelson-Oppen method. As far as theory solver combination is concerned, our approach and that in [6] are in a sense dual, possibly as a consequence of their different motivations. Simplifying a bit, in [6] the theory solvers are (or can be) completely unaware of each other. The DPLL engine is in charge of identifying shared constants and feeding (dis)equalities between them as appropriate to the solvers. This way, off-theshelf decision procedures can be used as theory solvers. In our case, the roles are reversed. As we will see, the solvers are aware of their shared constants, and are in charge of producing lemmas containing (dis)equalities between them, for the engine to split on. The advantage in this case is that the same mechanism already in place for splitting on demand can be used for combination as well, with no changes to the engine.

\section{Extended Abstract DPLL Modulo Theories}

In this section, we briefly describe the Abstract DPLL Modulo Theories framework (see [12] for more details) and then extend it so that it can be used to formalize our new version of $\operatorname{DPLL}(T)$ and, more generally, SMT approaches where new atoms and new symbols are introduced.

\subsection{Abstract DPLL Modulo Theories}

As usual in SMT, given a theory $T$ (a set of closed first-order formulas), we will only consider the SMT problem for ground (and hence quantifier-free) CNF formulas $F$. Such formulas may contain free constants, i.e., constant symbols not in the signature of $T$, which, as far as satisfiability is concerned, can be equivalently seen as existential variables. Other than free constants, all other predicate and function symbols in the formulas will instead come from the signature of $T$. From now on, we will assume that all formulas satisfy these restrictions.

The formalism we describe is based on a set of states together with a binary relation $\Longrightarrow$ (called the transition relation) over these states, defined by means of transition rules. Starting with a state containing an input formula $F$, one can use the rules to generate a finite sequence of states, where the final state indicates whether or not $F$ is $T$-consistent. 
A state is either the distinguished state FailState (denoting T-unsatisfiability) or a pair of the form $M \| F$, where $M$ is a sequence of literals, with $\emptyset$ denoting the empty sequence, and $F$ is a formula in conjunctive normal form (CNF), i.e., a finite set of disjunctions of literals. We additionally require that $M$ never contains both a literal and its negation and that each literal in $M$ is annotated as either a decision literal (indicated by $l^{\mathrm{d}}$ ) or not. Frequently, we will refer to $M$ as a partial assignment or consider $M$ just as a set or conjunction of literals, ignoring both the annotations and the order of its elements.

In what follows, a possibly subscripted or primed lowercase $l$ always denotes a literal. Similarly $C$ and $D$ always denote clauses (disjunctions of literals), $F$ and $G$ denote conjunctions of clauses, and $M$ and $N$ denote partial assignments.

We write $M \mid=F$ to indicate that $M$ propositionally satisfies $F$. If $C$ is a clause $l_{1} \vee \ldots \vee l_{n}$, we sometimes write $\neg C$ to denote the formula $\neg l_{1} \wedge \ldots \wedge \neg l_{n}$. We say that $C$ is conflicting in a state $M \| F, C$ if $M \models \neg C$.

A formula $F$ is called $T$-(in) consistent if $F \wedge T$ is (un)satisfiable in the firstorder sense. We say that $M$ is a T-model of $F$ if $M \models F$ and $M$, seen as a conjunction of literals, is $T$-consistent. It is not difficult to see that $F$ is $T$ consistent if, and only if, it has a $T$-model. If $F$ and $G$ are formulas, then $F$ entails $G$ in $T$, written $F \models_{T} G$, if $F \wedge \neg G$ is $T$-inconsistent. If $F \models_{T} G$ and $G \models_{T} F$, we say that $F$ and $G$ are $T$-equivalent. A theory lemma is a clause $C$ such that $\emptyset=_{T} C$.

We start with the transition system first presented in [12]. ${ }^{2}$

Definition 1. Abstract DPLL Modulo Theories consists of the following rules:

\section{UnitPropagate :}

$$
M\|F, C \vee l \Longrightarrow M l\| F, C \vee l \text { if }\left\{\begin{array}{l}
M \models \neg C \\
l \text { is undefined in } M
\end{array}\right.
$$

Decide :

$$
M\left\|F \quad \Longrightarrow M l^{\mathrm{d}}\right\| F \quad \text { if }\left\{\begin{array}{l}
l \text { or } \neg l \text { occurs in a clause of } F \\
l \text { is undefined in } M
\end{array}\right.
$$

Fail :

$$
M \| F, C \quad \Longrightarrow \text { FailState } \quad \text { if }\left\{\begin{array}{l}
M \models \neg C \\
M \text { contains no decision literals }
\end{array}\right.
$$

T-Learn :

$$
M\|F \quad \Longrightarrow M\| F, C \quad \text { if }\left\{\begin{array}{l}
\text { each atom of } C \text { occurs in } F \text { or in } M \\
F \models_{T} C
\end{array}\right.
$$

T-Backjump :

$$
M l^{\mathrm{d}} N\left\|F, C \Longrightarrow M l^{\prime}\right\| F, C \text { if }\left\{\begin{array}{l}
M l^{\mathrm{d}} N \models \neg C \text {, and there is } \\
\text { some clause } C^{\prime} \vee l^{\prime} \text { such that: } \\
F, C \models_{T} C^{\prime} \vee l^{\prime} \text { and } M \models \neg C^{\prime}, \\
l^{\prime} \text { is undefined in } M, \text { and } \\
l^{\prime} \text { or } \neg l^{\prime} \text { occurs in } F \text { or in } M l^{\mathrm{d}} N
\end{array}\right.
$$

${ }^{2}$ For simplicity, we omit the Restart and $T$-Forget rules. A complete treatment of these rules is included in the full report [2]. 
T-Propagate :

$$
M\|F \quad \Longrightarrow M l\| F \text { if }\left\{\begin{array}{l}
M \models_{T} l \\
l \text { or } \neg l \text { occurs in } F \\
l \text { is undefined in } M
\end{array}\right.
$$

The Basic DPLL Modulo Theories system consists of the rules Decide, Fail, UnitPropagate, T-Propagate and T-Backjump. We denote the transition relation defined by these rules by $\Longrightarrow_{\mathrm{B}}$. We denote the transition relation defined by all the rules by $\Longrightarrow_{\mathrm{FT}}$.

For a transition relation $\Longrightarrow$, we denote by $\Longrightarrow^{*}$ the reflexive-transitive closure of $\Longrightarrow$. We call any sequence of the form $S_{0} \Longrightarrow S_{1}, S_{1} \Longrightarrow S_{2}, \ldots$ a derivation, and denote it by $S_{0} \Longrightarrow S_{1} \Longrightarrow S_{2} \Longrightarrow \ldots$. We call any subsequence of a derivation a subderivation. If $S \Longrightarrow S^{\prime}$ we say that there is a transition from $S$ to $S^{\prime}$. A state $S$ is final with respect to $\Longrightarrow$ if there are no transitions from $S$.

The relevant derivations in the Abstract DPLL Modulo Theories system are those that start with a state of the form $\emptyset \| F$, where $F$ is a formula to be checked for $T$-consistency, and end in a state that is final with respect to $\Longrightarrow_{\mathrm{B}}$.

\subsection{The Extended Abstract DPLL Modulo Theories System}

Any realization of the Abstract DPLL Modulo Theories framework, in addition to implementing the rules and an execution strategy, must be able to determine the $T$-consistency of $M$ when a final state $M \| F$ is reached. For this purpose, one typically assumes the existence of Solver $_{T}$ which can do precisely that.

However, for some important theories, determining the $T$-consistency of a conjunction of literals requires additional internal case splitting. In order to simplify Solver $_{T}$ and centralize the case splitting in the DPLL engine, it is desirable to relax the requirement on Solver $_{T}$ by allowing it to demand that the DPLL engine do additional case splits before determining the $T$-consistency of the partial assignment. For flexibility - and because it is needed by actual theories of interest - the theory solver should be able to demand case splits on literals that do not appear in $M$ or $F$ and possibly even contain fresh constant symbols.

It is not hard to see, however, that allowing this kind of flexibility poses a potential termination problem. We can overcome this difficulty if, for any input formula $F$, the set of all literals needed to check the $T$-consistency of $F$ is finite. More precisely, as a purely theoretical construction, we assume that for every input formula $F$ there is a finite set $\mathcal{L}(F)$ of literals containing all literals on which a given theory solver may demand case splits when starting with a conjunction of literals from $F$. For example, for a solver for the theory of arrays $\mathcal{L}(F)$ could contain atoms of the form $i=j$, where $i$ and $j$ are array indices occurring in $F$. This technical requirement poses no limitations on any of the practically useful theory solver procedures we are aware of (see Section 3). Also, for the proofs here there is no need to construct the set $\mathcal{L}(F)$. It is enough to know that it exists. Formally, we require the following. 
Definition 2. $\mathcal{L}$ is a suitable literal-generating function if for every finite set of literals $L$ :

1. $\mathcal{L}$ maps $L$ to a new finite set of literals $L^{\prime}$ such that $L \subseteq L^{\prime}$.

2. For each atomic formula $\alpha, \alpha \in \mathcal{L}(L)$ iff $\neg \alpha \in \mathcal{L}(L)$.

3. If $L^{\prime}$ is a set of literals and $L \subseteq L^{\prime}$, then, $\mathcal{L}(L) \subseteq \mathcal{L}\left(L^{\prime}\right)$ (monotonicity).

4. $\mathcal{L}(\mathcal{L}(L))=\mathcal{L}(L)$ (idempotence).

For convenience, given a formula $F$, we denote by $\mathcal{L}(F)$ the result of applying $\mathcal{L}$ to the set of all literals appearing in $F$.

The introduction of new constant symbols poses potential problems not only for termination, but also for soundness. One property of the transition relation $\Longrightarrow_{\mathrm{FT}}$ is that whenever $\emptyset\left\|F \Longrightarrow{ }_{\mathrm{FT}}^{*} M\right\| F^{\prime}$, the formulas $F$ and $F^{\prime}$ are $T$-equivalent. This will no longer be true if we allow the introduction of new constant symbols. However, it is sufficient to simply ensure $T$-equisatisfiability of $F$ and $F^{\prime}$. To this end, we introduce the following definition.

Definition 3. Given a formula $F$ and a formula $G$, we define $\gamma_{F}(G)$ as follows:

1. Let $G^{\prime}$ be the formula obtained by replacing each free constant symbol in $G$ that does not appear in $F$ with a fresh variable.

2. Let $\bar{v}$ be the set of all fresh variables introduced in the previous step.

3. Then, $\gamma_{F}(G)=\exists \bar{v}$. $G^{\prime}$.

Now we can give a new transition rule called Extended $T$-Learn which replaces $T$-Learn and allows for the desired additional flexibility.

Definition 4. The Extended DPLL Modulo Theories system, denoted as $\Longrightarrow \mathrm{xT}$, consists of the rules of Basic DPLL Modulo Theories, together with the rule:

Extended T-Learn

$M\|F \quad \Longrightarrow M\| F, C$ if $\left\{\begin{array}{l}\text { each atom of } C \text { occurs in } F \text { or in } \mathcal{L}(M) \\ F \models_{T} \gamma_{F}(C)\end{array}\right.$

The key observation is that an implementation using Extended $T$-Learn has more flexibility when a state $M \| F$ is reached which is final with respect to $\Longrightarrow_{\mathrm{B}}$. Whereas before it would have been necessary for the theory solver to determine the $T$-consistency of $M$ when such a state was reached, the Extended $T$-Learn rule allows the possibility of delaying a response by demanding that additional case splits (on possibly new literals appearing in the clause $C$ ) be done first. As we will show below, the properties of $\mathcal{L}$ ensure that the solver's response cannot be delayed indefinitely.

\subsection{Correctness of Extended Abstract DPLL Modulo Theories}

A decision procedure for SMT can be obtained by generating a derivation using $\Longrightarrow$ XT with a particular strategy. As with $\Longrightarrow_{\mathrm{FT}}$, the aim of a derivation is to compute a state $S$ such that: (i) $S$ is final with respect to the rules of Basic DPLL Modulo Theories and (ii) if $S$ is of the form $M \| F$ then $M$ is $T$-consistent. 
Lemma 1. If $\emptyset\left\|F \Longrightarrow{ }_{\mathrm{XT}}^{*} M\right\| G$ then the following hold.

1. All the literals in $M$ and all the literals in $G$ are in $\mathcal{L}(F)$.

2. $M$ contains no literal more than once and is indeed an assignment, i.e., it contains no pair of literals of the form $p$ and $\neg p$.

3. $G \models_{T} F$ and for some $H, F \models_{T} \gamma_{H}(G)$.

4. If $M$ is of the form $M_{0} l_{1} M_{1} \ldots l_{n} M_{n}$, where $l_{1}, \ldots, l_{n}$ are all the decision literals of $M$, then $G, l_{1}, \ldots, l_{i} \models_{T} M_{i}$ for all $i$ in $0 \ldots n$.

Theorem 1 (Termination of $\Longrightarrow \mathrm{xT}$ ). There is no infinite derivation of the form $\emptyset \| F \Longrightarrow \mathrm{xT} S_{1} \Longrightarrow \mathrm{xT} \ldots$

The main difference in the termination argument with respect to $\Longrightarrow_{\mathrm{FT}}$ is that, while Extended $T$-Learn can produce lemmas with new literals, it can only produce a finite number of them thanks to the properties of $\mathcal{L}$.

Lemma 2. If $\emptyset\left\|F \Longrightarrow{ }_{\mathrm{XT}}^{*} M\right\| F^{\prime}$ and there is some conflicting clause in $M \| F^{\prime}$, i.e., $M \models \neg C$ for some clause $C$ in $F^{\prime}$, then either Fail or T-Backjump applies to $M \| F^{\prime}$.

Property 1. If $\emptyset\left\|F \Longrightarrow{ }_{\mathrm{XT}}^{*} M\right\| F^{\prime}$ and $M$ is $T$-inconsistent, then either there is a conflicting clause in $M \| F^{\prime}$, or else Extended $T$-Learn applies to $M \| F^{\prime}$, generating a clause enabling some Basic DPLL Modulo Theories step.

Lemma 2 and Property 1 show that, for a state of the form $M \| F$, if there is some literal of $F$ undefined in $M$, or there is some conflicting clause, or $M$ is $T$-inconsistent, then a rule of Basic DPLL Modulo Theories is always applicable, possibly after a single Extended $T$-Learn step. Together with Theorem 1 (Termination), this shows how to compute a state to which the following main theorem is applicable.

Theorem 2. Let Der be a derivation $\emptyset \| F \Longrightarrow{ }_{\mathrm{XT}}^{*} S$, where $S$ is (i) final with respect to Basic DPLL Modulo Theories, and (ii) if $S$ is of the form $M \| F^{\prime}$ then $M$ is T-consistent. Then

1. $S$ is FailState if, and only if, $F$ is T-inconsistent.

2. If $S$ is of the form $M \| F^{\prime}$ then $M$ is a T-model of $F$.

For a given theory $T$, Theorems 1 and 2 show how to obtain a decision procedure for the $T$-consistency of formulas as long as we have a theory solver and can prove for it the existence of a suitable literal-generating function $\mathcal{L}$ such that the following holds: for every state of the form $M \| F$ that is final with respect to $\Longrightarrow_{\mathrm{B}}$, the theory solver is able to (i) determine that $M$ is $T$ inconsistent, (ii) determine that $M$ is $T$-consistent, or (iii) generate a new clause via Extended T-Learn that enables some Basic DPLL Modulo Theories step. 


\section{Avoiding case splitting within theory solvers}

In this section, we show how rule-based theory solvers can be used in the context of Extended DPLL Modulo Theories.

Recall that theory solvers only need to deal with conjunctions (equivalently, sets) of literals. Then observe that any solver deciding the $T$-consistency of such conjunctions in a theory where this problem is NP-hard is bound to resort to some form of case splitting. ${ }^{3}$ We show how the Extended $T$-Learn rule allows such solvers to avoid any internal case splitting, and we explain why, for rule-based solvers, the existence of $\mathcal{L}$ is reasonable.

\subsection{Rule-based Theory Solvers}

A large class of theory solvers can be defined using inference rules that describe how to take a set of literals and transform it in some way to get new sets of literals (or $\perp$, indicating $T$-inconsistency). Consider a theory $T$. For our purposes, let us assume that an inference rule has one of the following two formats:

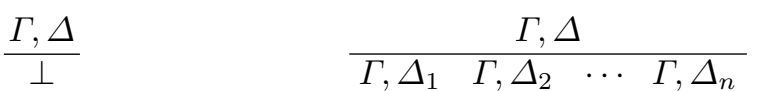

where the meta-variables $\Gamma, \Delta$ and $\Delta_{i}$ represent sets of literals. We call rules of the first kind refuting rules and rules of the second kind progress rules. Typically, $\Delta$ has side-conditions or is a schema, while $\Gamma$ can represent any set of literals. Progress rules describe a local change based on a small number of literals (the ones in $\Delta$ ), while all of the other literals (the ones in $\Gamma$ ) are unchanged.

A refuting rule is sound iff any legal instance $\delta$ of $\Delta$ is $T$-inconsistent. A progress rule is sound if whenever $\Delta, \Delta_{1}, \ldots, \Delta_{n}$ are instantiated with $\delta, \delta_{1}, \ldots, \delta_{n}$ respectively, $\delta$ is $T$-consistent iff $\bigvee_{i=1}^{n} \delta_{i}$ is $T$-consistent. We say that a set $\Phi$ of literals is (ir)reducible with respect to a set of derivation rules $R$ if (n)one of the rules in $R$ applies to it, i.e., if (no) some subset of $\Phi$ is a legal instance of $\Delta$ in a rule of $R$. A strategy is a function that, given a reducible set of literals $\Phi$, chooses a rule from $R$ to apply.

Given a set $R$ of rules and a strategy $S$, a derivation tree for a set of literals $\Phi$ is a finite tree with root $\Phi$ such that for each internal node $E$ of the tree, $E$ is reducible and its children are the conclusions of the rule selected by $S$ for $E$. A refutation tree (for $\Phi$ ) is a derivation tree all of whose leaves are $\perp$. A derivation is a sequence of derivation trees starting with the single-node tree containing $\Phi$, where each tree is derived from the previous one by the application of a rule from $R$ to one of its leaves. A refutation is a finite derivation ending with a refutation tree. A strategy $S$ is terminating if every derivation using $S$ is finite. A strategy $S$ is complete if whenever $\Phi$ is $T$-inconsistent, $S$ produces a refutation for $\Phi$.

It is not hard to see that a set $R$ of sound inference rules together with a terminating and complete strategy $S$ provide a decision procedure for the $T$ consistency of sets of ground literals. In fact, all decision procedures typically

\footnotetext{
${ }^{3}$ In fact, conceivably a solver may be based on case splitting even if the above $T$ consistency problem is polynomial, for simplicity or convenience.
} 
associated with applications of Satisfiability Modulo Theories can be described in this way. We will now describe how such decision procedures can be incorporated into the Extended Abstract DPLL Modulo Theories formalism.

\subsection{Integration with Rule-based Theory Solvers}

Recall that the original DPLL Modulo Theories framework requires that for every state $M \| F$ that is final with respect to Basic DPLL Modulo Theories, the theory solver can determine the $T$-consistency of $M$. Given a set of sound inference rules and a terminating and complete strategy, $M$ can be checked for $T$ consistency simply by generating the derivation starting with $M$ and determining whether it results in a refutation tree or not.

Note that this process may require a large derivation tree with many branches. The purpose of the Extended T-Learn rule is to allow the theory solver to avoid having to do any splitting itself. This can be accomplished as follows. Given a state $M \| F$ which is final with respect to Basic DPLL Modulo Theories, the theory solver begins applying rules starting with $M$. However, this time, as soon as a splitting rule is encountered (a progress rule with $n>1$ ), the theory solver halts and uses Extended $T$-Learn to return one or more clauses representing the case split. The theory solver is then suspended until another final state $M^{\prime} \| F^{\prime}$ is reached.

The obvious remaining question is how to capture the case split with a learned clause. As we show in [2], one way to do this that will work for any rule-based theory solver is to encode the number of possible case splits using Boolean constants. In practice, however, it is usually possible and desirable to encode splitting rules more directly. For example, a progress rule of the form seen in the previous subsection (where $n>1$ ) corresponds to the following formula schema: $\neg(\Delta) \vee \bigvee_{i=1}^{n} \Delta_{i}$. Any instance of this schema can be converted into CNF and the resulting clauses sent to the DPLL engine via Extended $T$-Learn. For this to work, one additional requirement is that the rules be refining. We say that an inference rule is refining if it is a refuting rule or if whenever $\Delta, \Delta_{1}, \ldots, \Delta_{n}$ are instantiated with $\delta, \delta_{1}, \ldots, \delta_{n}$ respectively, $\delta \models_{T} \gamma_{\delta}\left(\bigvee_{i=1}^{n} \delta_{i}\right)$. This is essentially a stronger version of soundness. It requires that any model of the premise can be refined into a model of some of the consequents. It is necessary in order to satisfy the side conditions of Extended $T$-Learn.

We must also check that an appropriate literal-generating function $\mathcal{L}$ exists. Assume we are given a set $R$ of rules and a terminating strategy $S$. First, define $\mathcal{D}$ to be a function which, given a set $\Phi$ of literals returns all literals that may appear along any branch of the derivation tree with any subset of $\Phi$ at its root. And let $\mathcal{N}$ be a function which, given a set $\Phi$ of literals, returns all literals that can be formed from the atomic formulas in $\Phi$. Now, we define a series of functions $\mathcal{L}_{i}$ as follows. Let $\mathcal{L}_{0}$ be the identity function and for $i>0$, let $\mathcal{L}_{i}(\Phi)=\mathcal{N}\left(\mathcal{D}\left(\mathcal{L}_{i-1}(\Phi)\right)\right)$. If for some $k>0, \mathcal{L}_{k}=\mathcal{L}_{k+1}$, then we say that $R$ is literal-bounded under $S$, and define $\mathcal{L}=\mathcal{L}_{k}$. 
Property 2. If $R$ is a set of sound refining rules for a theory $T, S$ is a strategy for $R$ that is terminating and complete, and $R$ is literal-bounded under $S$, then $R$ can be integrated with the Extended DPLL Modulo Theories framework.

Proof. We first show that $\mathcal{L}$ satisfies Definition 2. It is easy to see that Properties 1 and 2 in the definition are satisfied. Because $\mathcal{D}(\Phi)$ considers derivations starting with any subset of $\Phi$, Property 3 must also be satisfied. Finally, because $\mathcal{L}$ is a fixed point of $\mathcal{L}_{i}$, it must be idempotent.

Now, we must show that whenever a state $M \| F$ is reached that is final with respect to Basic DPLL Modulo Theories, the theory solver can do one of the following: determine that $M$ is $T$-consistent; determine that $M$ is $T$-inconsistent; or introduce a new clause via Extended T-Learn that enables some Basic DPLL Modulo Theories step.

Given a state $M \| F$, we simply apply rules from $R$ to $M$ according to strategy $S$. If $\perp$ is derived, then by soundness, $M$ is $T$-inconsistent. If an irreducible set of literals is derived, then by completeness, $M$ must be $T$-consistent. If a splitting rule is reached, and $\Gamma, \Delta, \Delta_{1}, \ldots, \Delta_{n}$ are instantiated with $\phi, \delta, \delta_{1}, \ldots, \delta_{n}$ respectively, there are three possibilities:

1. For all $i, M \models \neg \delta_{i}$. In this case, we apply Extended $T$-Learn to learn $\neg(\delta) \vee$ $\bigvee_{i=1}^{n} \delta_{i}$, which will result in one or more clauses that are conflicting in $M$, thus enabling either Fail or T-Backjump by Lemma 2.

2. For some $i, M \models \delta_{i}$, or else $\delta_{i}$ is undefined in $M$ and $M \models \neg \delta_{j}$ for every $j \neq i$. In either case, no split is necessary and we simply proceed by applying rules of $R$ to $\phi, \delta_{i}$.

3. The final case is when at least two of the $\delta_{i}$ are undefined in $M$. Then we apply Extended $T$-Learn to learn $\neg(\delta) \bigvee \bigvee_{i=1}^{n} \delta_{i}$ which is guaranteed to contain at least one clause that is not satisfied by $M$, thus enabling Decide.

Example 3. As we saw in a previous example the theory of arrays requires case splitting. One (sound and refining) rule-based decision procedure for this theory is given in [13]. A careful examination of the decision procedure reveals the following: (i) each term can be categorized as an array term, an index term, a value term, or a set term; (ii) no new array terms are ever introduced by the inference rules; (iii) at most one new index term for every pair of array terms is introduced; (iv) set terms are made up of some finite number of index terms; (v) the only new value terms introduced are of the form $\operatorname{read}(a, i)$ where $a$ is an array term and $i$ is an index term. It follows that the total number of possible terms that can be generated by the procedure starting with any finite set of literals is finite. Because there are only a finite number of predicates, it then follows that this set of rules is literal-bounded.

\section{Application to Satisfiability Modulo Multiple Theories}

In this section, we focus on background theories $T$ that are actually the union of two or more component theories $T_{1}, \ldots, T_{n}$, each equipped with its own solver. 
We first show how to obtain an Abstract DPLL Modulo Theories transition system for the combined theory $T$ as a refinement of the system XT described in Section 2 using only the solvers of the theories $T_{i}$. Then we show how to refine the new $\operatorname{DPLL}(T)$ architecture into a $\operatorname{DPLL}\left(T_{1}, \ldots, T_{n}\right)$ architecture in which each $T_{i}$-solver is directly integrated into the $\operatorname{DPLL}\left(X_{1}, \ldots, X_{n}\right)$ engine.

We will work here in the context of first-order logic with equality. For the rest of the section we fix $n>1$ stably infinite theories ${ }^{4} T_{1}, \ldots, T_{n}$ with respective, mutually disjoint signatures $\Sigma_{1}, \ldots, \Sigma_{n}$. We will consider the theory $T=T_{1} \cup$ $\cdots \cup T_{n}$ with signature $\Sigma=\Sigma_{1} \cup \cdots \cup \Sigma_{n}$. We are interested in the $T$-satisfiability of ground formulas over the signature $\Sigma$ extended with an infinite set $K$ of free constants. For any signature $\Omega$ we will denote by $\Omega(K)$ the signature $\Omega \cup K$. We say that a ground clause or literal is $(i-)$ pure if it has signature $\Sigma_{i}(K)$ where $i \in\{1, \ldots, n\}$. Given a CNF formula $F$ of signature $\Sigma(K)$, by abstracting subterms with fresh constants from $K$, it is possible to convert $F$ in linear time into an equisatisfiable CNF formula, all of whose atoms are pure. See [14], for instance, for details on this purification procedure. From now on, we will limit ourselves with no loss of generality to pure formulas.

Following the Nelson-Oppen combination method, the various solvers will cooperate by exchanging entailed equalities over shared constants. Let $L$ be a set of pure literals over the signature $\Sigma(K)$. We say that a constant $k \in K$ is an (ij-)shared constant of $L$ if it occurs in an $i$-pure and a $j$-pure literal of $L$ for some distinct $i$ and $j$. For $i=1, \ldots, n$, we denote by $L^{i}$ the set of all the $\Sigma_{i}(K)$-literals of $L$ and by $\mathcal{S}_{i}(L)$ the set of all equalities between distinct $i j$ shared constants of $L$ for every $j \neq i$. Note that for every $j \neq i, L^{j} \cap L^{i}$ contains at most equalities or the negation of equalities from $\mathcal{S}_{i}(L)$. An arrangement for $L$ is a set containing for each equality $e \in \bigcup_{i} \mathcal{S}_{i}(L)$ either $e$ or $\neg e$ (but not both), and nothing else.

The extended Abstract DPLL Modulo theories framework can be refined to take into account that $T$ is a combined theory by imposing the following additional requirements on the XT system.

Refinement 1 . We consider only derivations starting with states of the form $\emptyset \| F$, where each atom of $F$ is a pure $\Sigma(K)$-atom.

Refinement 2. We consider only applications $M\|F \Longrightarrow M l\| F$ of $T$-Propagate and applications $M\|F \Longrightarrow M\| F, C$ of Extended $T$-Learn where $l$ and each literal of $C$ are pure.

Refinement 1 and 2 maintain the invariant that all the literals occurring in a state are pure, and so can be fed to the corresponding local solvers. Given these minimal requirements, it will be sufficient for $T$-Propagate to propagate only literals $l$ that are $i$-pure for some $i=1, \ldots, n$ and such that and $\left.M^{i}\right|_{T_{i}}$ $l$, where the entailment $M^{i} \models_{T_{i}} l$ is determined by the $T_{i}$-solver. Similarly, Extended $T$-Learn will rely on the local solvers only to learn $T_{i}$-lemmas, i.e., $i$-pure clauses $C$ such that $\emptyset \models_{T_{i}} \gamma_{F}(C)$. Note that we do allow lemmas $C$ consisting

\footnotetext{
${ }^{4}$ A theory $T$ is stably infinite if every $T$-consistent quantifier-free formula $F$ over $T$ 's signature is satisfiable in an infinite model of $T$.
} 
of pure literals from different theories and such that $F \models_{T} \gamma_{F}(C)$, as lemmas of this sort can be computed even if one only has local solvers (consider for example the backjump clauses generated by standard conflict analysis mechanisms).

Refinement 3. The suitable literal-generating function $\mathcal{L}$ maps every finite set $L$ of pure $\Sigma(K)$-literals to a finite set of pure $\Sigma(K)$-literals including $\bigcup_{i} \mathcal{S}_{i}(L)$.

To use the various solvers together in a refutationally complete way for $T$ consistency, it is necessary to make them agree on an arrangement. To do this efficiently, they should be able to share the entailed (disjunctions) of equalities of shared constants. Refinement 3 then essentially states that theory lemmas can include shared equalities.

\subsection{From $\operatorname{DPLL}(T)$ to $\operatorname{DPLL}\left(T_{1}, \ldots, T_{n}\right)$}

Assuming the previous refinements at the abstract level, we now show in concrete how the $\operatorname{DPLL}(T)$ architecture can be specialized to use the various local solvers directly and to facilitate cooperation among them. Here we define a local requirement on each $T_{i}$-solver that does not even need refutational completeness for $T_{i}$-consistency. If $M$ is an assignment consisting of pure literals and $i \in\{1, \ldots, n\}$, we call the (default) completion of $M^{i}$ and denote by $\widehat{M}^{i}$ the smallest extension of $M^{i}$ falsifying every shared equation for $M^{i}$ that is undefined in $M$, that is, $\widehat{M}^{i}=M^{i} \cup\left\{\neg e \mid e \in \mathcal{S}_{i}(M), e\right.$ undefined in $\left.M\right\}$.

Requirement 1. For each $i=1, \ldots, n$, the solver for $T_{i}$, given a state $M \| F$, must be able to do one of the following:

1. determine that $\widehat{M}^{i}$ is $T_{i}$-consistent, or

2. identify a $T_{i}$-inconsistent subset of $M^{i}$, or

3. produce an $i$-pure clause $C$ containing at least one literal of $\mathcal{L}(M)$ undefined in $M$ and such that $\emptyset \models_{T_{i}} \gamma_{F}(C)$.

The computational cost of the test in Point 1 of this requirement depends on the deduction capabilities of the theory solver. ${ }^{5}$ Note, however, that the test can be deferred thanks to Point 3. The solver may choose not to generate the completion of $M^{i}$ explicitly and test it for $T_{i}$-inconsistency, and instead generate a lemma for the engine containing one of the undefined equalities.

If a solver meeting Requirement 1 cannot determine the $T_{i}$-consistency of the completion $\widehat{M}^{i}$, it must be either because it has determined that a subset of $\widehat{M}^{i}$ (possibly of $M^{i}$ alone) is in fact inconsistent, or that it needs more information about some of the undefined literals of $\mathcal{L}(M)$ first. However, once every literal of $\mathcal{L}(M)$ is defined in $M$, including the equalities in $\mathcal{S}_{i}(M)$, the solver must be able to tell whether $M^{i}$ is $T_{i}$-consistent or not. This is a minimal requirement for any solver to be used in a Nelson-Oppen style combination procedure.

Usually though it is desirable for Nelson-Oppen solvers to also be able to compute (disjunctions of) shared equalities entailed by a given set of literals, so

\footnotetext{
${ }^{5}$ For some solvers, such as the common ones for EUF or linear rational arithmetic, this additional cost is actually zero as these solvers already explicitly maintain complete information on all the entailed equalities between the known terms.
} 
that only these equalities can be propagated to the other solvers, and guessing is minimized. For instance, if one solver communicates that $a$ is equal to either $b$ or $c$, then the other solvers do not have to consider cases where $a$ is equal to some fourth constant. Requirement 1 allows that possibility, as illustrated by the following example.

Example 4. Assume, just for simplicity, that for every $M, \mathcal{L}(M)$ is no more than $M \cup \bigcup_{i} \mathcal{S}_{i}(M)$, which entails that each $T_{i}$-solver is refutationally complete for $T_{i}$-consistency. Then consider an assignment $M$ where $M_{i}$ is $T_{i}$-consistent, for some $i$, and let $e_{1}, \ldots, e_{n}$ be equalities in $\mathcal{S}_{i}(M)$ undefined in $M$ such that $l_{1}, \ldots, l_{m} \models \bigvee_{k} e_{k}$ for some $\left\{l_{1}, \ldots, l_{m}\right\} \subseteq M^{i}$. In this case, $\widehat{M}^{i}$ is clearly $T_{i^{-}}$ inconsistent. However, since $M^{i}$ alone is consistent, by Requirement 1 the $T_{i^{-}}$ solver must return a lemma containing one or more undefined literals of $\mathcal{L}(M)$.

Now, if the solver can in fact compute (deterministically, with no internal case splits!) the clause $\bigwedge_{j} l_{j} \Rightarrow \bigvee_{k} e_{k}$, that clause will be the ideal lemma to return. Otherwise, it is enough for the solver to return any lemma that contains at least one shared equality $e$ (in the worst case, even a tautology of the form $e \vee \neg e$ will do). Intuitively, this marks a progress in the computation because eventually one of the shared equalities will be added to $M$ (for instance, by an application of Decide), reducing the number of undefined literals in $\mathcal{L}(M)$.

Requirement 1 and the earlier refinements are enough to guarantee that we can use the local $T_{i}$-solvers directly - as opposed to building a solver for the combined theory $T$ - to generate derivations satisfying Theorem 2 . The first thing we need for Theorem 2 is easy to see: it is always possible to derive from a state $\emptyset \| F$ a final state $S$ with respect to Basic DPLL Modulo Theories $\left(\Longrightarrow_{\mathrm{B}}\right)$. The second thing we need is that whenever the final state $S$ has the form $M \| F^{\prime}$, the assignment $M$ is $T$-consistent. Although none of the local solvers is able to determine that by itself, it can do it in cooperation with the other solvers thanks to Requirement 1. It is then not difficult to show (see [2]) that, under the assumptions in this section, the following property holds.

Property 3. Each derivation of the form $\emptyset\left\|F \Longrightarrow \mathrm{XT}^{*} M\right\| G$ where $M \| G$ is final wrt. Basic DPLL Modulo Theories can be extended in finitely many steps using the solvers for $T_{1}, \ldots, T_{n}$ to a derivation of the form $\emptyset\left\|F \Longrightarrow{ }_{\mathrm{XT}}^{*} M\right\| G \Longrightarrow{ }_{\mathrm{XT}}^{*} S$ where $S$ is either FailState or a state $M^{\prime} \| G^{\prime}$ with a $T$-consistent $M^{\prime}$.

\section{Conclusions and further work}

We have proposed a new version of $\operatorname{DPLL}(T)$ in which theory solvers can delegate all case splits to the DPLL engine. This can be done on demand for solvers that can encode their internal case splits into one or more clauses, possibly including new constants and literals. We have formalized this in an extension of Abstract DPLL and proved it correct. We think that the new insights gained by this formalization will help us and others when incorporating these ideas into our respective SMT solvers. 
We have also introduced a $\operatorname{DPLL}\left(T_{1}, \ldots, T_{n}\right)$ architecture for combined theories, which also fits naturally into the extended Abstract DPLL framework. This refinement is crucial in practice because most SMT applications are based on combinations of theories.

Our splitting on demand approach leads to significantly simpler theory solvers. The price to pay is an increase in the complexity of the $\operatorname{DPLL}(X)$ engine, which must be able to deal with a dynamically expanding set of literals. However, we believe that doing this once and for all is better than paying the price of building a far more complex theory solver for each theory that requires case splits. Moreover, the requirement of being able to deal dynamically with new literals and clauses is needed in general for flexibility in applications, not just for our approach.

As future work, we plan to evaluate the approach experimentally. We also plan to investigate theory-dependent splitting heuristics, and effective ways for a theory solver to share such heuristic information with the $\operatorname{DPLL}(X)$ engine.

\section{References}

1. C. Barrett, L. de Moura, and A. Stump. SMT-COMP: Satisfiability Modulo Theories Competition. In $C A V^{\prime} 05$, LNCS 3576, pages 20-23. Springer, 2005.

2. C. Barrett, R. Nieuwenhuis, A. Oliveras, and C. Tinelli. Splitting on demand in satisfiability modulo theories. Technical report. University of Iowa, 2006. Available at ftp://ftp.cs.uiowa.edu/pub/tinelli/papers/BarNOT-RR-06.pdf.

3. C. W. Barrett. Checking Validity of Quantifier-Free Formulas in Combinations of First-Order Theories. PhD thesis, Stanford University, 2003.

4. C. W. Barrett and S. Berezin. CVC lite: A new implementation of the cooperating validity checker. In $C A V^{\prime} 04$, LNCS 3114, pages 515-518. Springer, 2004.

5. C. Barrett, D. Dill, and A. Stump. Checking satisfiability of first-order formulas by incremental translation into SAT. In CAV'02, LNCS 2404, pages 236-249, 2002.

6. M. Bozzano, R. Bruttomesso, A. Cimatti, T. A. Junttila, S. Ranise, P. van Rossum, and R. Sebastiani. Efficient theory combination via boolean search. Information and Computation. To appear. Cf. conference paper at CAV'05.

7. D. Cantone and C. G. Zarba. A new fast tableau-based decision procedure for an unquantified fragment of set theory. In Automated Deduction in Classical and Non-Classical Logics, LNCS 1761, pages 127-137. Springer, 2000.

8. M. Davis, G. Logemann, and D. Loveland. A machine program for theoremproving. Comm. of the ACM, 5(7):394-397, 1962.

9. M. Davis and H. Putnam. A computing procedure for quantification theory. Journal of the ACM, 7:201-215, 1960.

10. H. Ganzinger, G. Hagen, R. Nieuwenhuis, A. Oliveras, and C. Tinelli. DPLL(T): Fast Decision Procedures. In $C A V^{\prime} 04$, LNCS 3114, pages 175-188. Springer, 2004.

11. G. Nelson and D. C. Oppen. Simplification by cooperating decision procedures. ACM Trans. Program. Lang. Syst., 1(2):245-257, 1979.

12. R. Nieuwenhuis, A. Oliveras, and C. Tinelli. Abstract DPLL and Abstract DPLL Modulo Theories. In LPAR'04, LNAI 3452, pages 36-50. Springer, 2005.

13. A. Stump, C. W. Barrett, D. L. Dill, and J. R. Levitt. A decision procedure for an extensional theory of arrays. In $L I C S^{\prime} 01$, pages 29-37. IEEE Computer Society.

14. C. Tinelli and M. T. Harandi. A new correctness proof of the Nelson-Oppen combination procedure. In FroCoS'96, pages 103-120. Kluwer Academic Publishers. 